\title{
Topological Non-connectivity Threshold in long-range spin systems
}

\author{
F. Borgonovi, ${ }^{1,2}$ G. L. Celardo, ${ }^{1}$ A. Musesti, ${ }^{1}$ R. Trasarti-Battistoni,${ }^{1}$ and P. Vachal ${ }^{3}$ \\ ${ }^{1}$ Dipartimento di Matematica e Fisica, Università Cattolica, via Musei 41, 25121 Brescia, Italy \\ 2 I.N.F.N., Sezione di Pavia, Italy \\ ${ }^{3}$ Faculty of Nucl. Sci. and Phys. Eng., Czech Technical University, Prague, Czech Republic
}

(Dated: June 4, 2018)

\begin{abstract}
We demonstrate the existence of a topological disconnection threshold, recently found in Ref. [1], for generic $1-d$ anisotropic Heisenberg models interacting with an inter-particle potential $R^{-\alpha}$ when $0<\alpha<1$ (here $R$ is the distance among spins). We also show that if $\alpha$ is greater than the embedding dimension $d$ then the ratio between the disconnected energy region and the total energy region goes to zero when the number of spins becomes very large. On the other hand, numerical simulations in $d=2,3$ for the long-range case $\alpha<d$ support the conclusion that such a ratio remains finite for large $N$ values. The disconnection threshold can thus be thought as a distinctive property of anisotropic long-range interacting systems.

PACS numbers: 05.50.+q, 75.10.Hk, 75.10.Pq
\end{abstract}

\section{INTRODUCTION}

Despite the wide use in statistical physics, long-range interacting systems, that is those systems characterized by a pairwise interaction decaying as a power law of the mutual distance with an exponent $\alpha$ less than the embedding dimension, do not have a well defined thermodynamic limit [2]. Also is it not at all clear whether their equilibrium properties can be described by the ordinary tools of statistical mechanics. For instance, the nonequivalence between the microcanonical and the canonical approach has been recently found in a long-range rotators model in the thermodynamic limit [3].

Besides these relevant implications in the foundation of statistical mechanics and in theoretical physics as well [4], the non-extensive behavior of long range systems has nowadays become important for applications too, ranging from neural systems $[\underline{5}$ to spin glasses $[\underline{6}$.

Within the class of long-range interacting systems, classical spin models, widely investigated during the last years [7], are the most easy-to-handle both from the analytical and the numerical point of view. Within such class of systems, (to be more precise, a class of anisotropic Heisenberg models) the existence of a threshold of disconnection in the energy surface has been demonstrated [1] for an interparticle interaction with infinite range. It has been called non-ergodicity threshold for historical reasons [8], even if the term can generate some confusion. Indeed non-ergodicity is only an obvious consequence: it simply means that the energy surface is topologically disconnected in two regions characterized by positive and negative magnetization. In other words it cannot exist a dynamical path connecting them and all trajectories starting from one region of the phase space stay there forever. For this reason we prefer here to call it Topological Non-connectivity Threshold (TNT).

The presence of the TNT cannot be considered an exotic mathematical peculiarity of some toy model. Its dynamical relevance has been studied in [9], where an explicit expression for the reversal times of the magnetiza- tion (the time necessary to jump from one branch to the other) has been given in the neighbors of the critical energy point. Reversal times diverge at the TNT as a power law with an exponent dependent on the number of the particles (and, probably, on the embedding dimension) as in ordinary phase transitions. Strictly speaking, even if in different context and for different models, the relationship between energy thresholds and topology transitions in the configuration space of classical spin models has been recently investigated [10].

Also, while the threshold was explicitly found within a class of anisotropic classical Heisenberg models with an easy axis of magnetization and all-to-all constant interaction, at the same time systems with nearest neighbor interaction were found to have a different behavior. For instance, the portion of disconnected energy region grows with the number of particles $N$, less than the energy itself, thus resulting in a zero ratio in the thermodynamic limit. Needless to say, such ratio stays finite for anisotropic coupling and all to all interaction.

While this feature is surely due to the anisotropy of the coupling (such finite ratio disappears for isotropic coupling even in the case of infinite range interaction), the question arises whether the presence of the TNT can be considered a pathological effect of the unphysical infinite interaction range or it is just somehow related with the long-range effects. This does not represent an academic question. Indeed, despite the possible applications of such model even in the case of all-to-all interaction 11], physical models require taking into account more realistic interactions, usually anisotropic 12 and depending generically from the inter-spin distance, as for the dipole-dipole coupling or when the spin is coupled with the electron spin of the conduction band of a metal, e.g. the RKKY model [13]. This leads quite naturally to Hamiltonians with an interparticle potential decaying as a generic power law with an exponent $\alpha$ of the relative distance $R$. The results found in Ref. 1] can thus be recovered by letting respectively $\alpha \rightarrow 0$ (all to all coupling) or $\alpha \rightarrow \infty$ (nearest neighbor coupling). 
Here, we extend the previous results to the whole class of models with an inverse power distance potential and show that $\alpha=1$ is a critical exponent for nonconnectivity in $d=1$ chains.

In general, the extension to higher dimensions is far from trivial, both numerically and analytically. However, we prove that the TNT, if any, can not "survive" (and we will specify the precise meaning below) when $N \rightarrow$ $\infty$, and $\alpha>d$. Numerical simulations in 2-d and $3-$ $\mathrm{d}$ also suggest that, for $\alpha<d$, the ratio between the disconnected energy region and the total energy range is finite in the thermodynamic limit. We thus conjecture that the TNT is a generic property of anisotropic longrange systems in any dimension.

\section{THE MODEL}

The Hamiltonian is a simple generalization of that considered in Ref. [1], and it is given by:

$$
H=-\frac{1}{2} \sum_{j \neq i}^{N} c_{|i-j|}\left(S_{i}^{y} S_{j}^{y}-\eta S_{i}^{x} S_{j}^{x}\right)
$$

where $\vec{S}_{i}=\left(S_{i}^{x}, S_{i}^{y}, S_{i}^{z}\right)$ is the spin vector with continuous components and modulus $1, N$ is the number of spins, $\eta(0 \leq \eta<1)$ is an anisotropic coefficient, and $c_{|i-j|}=|i-j|^{-\alpha}$, with $\alpha>0$. For definiteness we consider here only the case of an even number $N$ of classical spins.

Such kind of models are characterized by a minimal and maximal energy $E_{\min }, E_{\max }$, and by a finite energy range $E_{\text {max }}-E_{\text {min }}$ that we call energy spectrum (ES). In order to define properly the disconnection threshold, let us introduce the set $\mathcal{A}$ of all spin configurations with a zero projection of the total magnetization along the $y$-axis:

$$
\mathcal{A}=\left\{\mathcal{C}\left(\vec{S}_{1}, \ldots \vec{S}_{N}\right) \mid m_{y}=\sum_{i=1}^{N} S_{i}^{y}=0\right\}
$$

The TNT is thus defined as:

$$
E_{d i s}=\operatorname{Min}_{\mathcal{C} \in \mathcal{A}}[H],
$$

and the spin configurations corresponding to $E_{d i s}$ will be indicated as $\mathcal{C}_{\text {dis }}$. Here we are mainly interested in all those cases where the TNT, if any, occupies a significant portion of the ES in the thermodynamic limit. For this reason let us define the disconnection ratio:

$$
r=\frac{E_{d i s}-E_{\min }}{\left|E_{\min }\right|}>0
$$

A system will be considered disconnected only if $r \rightarrow$ const. $>0$, when $N \rightarrow \infty$. Note that the definition of $r$ given in Eq. (4) has a meaning only for systems with a bounded energy range.
A dynamical consequence of the TNT is that below it, a sample with a given initial magnetization $m_{y}$, cannot change the sign of $m_{y}$ for any time, since the constant energy surface is disconnected in a positive and a negative magnetization regions, thus no continuous dynamics can bring an isolated system from one region to the other.

Our proof will follow two steps: in the first part we find the minima of the $x$ and $y$ parts separately. Then we will show that the disconnected ratio goes to zero for longrange interaction, while it goes to some finite constant in the short range case.

\section{ONE DIMENSIONAL CASE}

\section{A. TNT, if any, is in the XY plane}

Roughly speaking, since Hamiltonian (11) is independent of the $z$-component of spins, the minimum will occur when the spins are as large as possible in (1), namely in the $X Y$ plane.

In order to prove that the configuration $\mathcal{C}_{\text {dis }}$ effectively lies in the $X Y$ plane, let us assume that it has some $S_{z}$ component different from zero. For definiteness assume $S_{1}^{z}>0$. It is then possible to define another configuration $\mathcal{C}^{\prime}$ simply making a rotation around the $y$-axis clockwise or counterclockwise which puts the spin $S_{1}^{z}$ onto the plane $X Y$. The energy difference between these two configurations can be computed at glance:

$$
\Delta E=\eta \sum_{i=2}^{N} c_{i-1} S_{i}^{x}\left( \pm \sqrt{1-\left(S_{1}^{y}\right)^{2}}-S_{1}^{x}\right) .
$$

Here the different sign \pm indicates the different way (clockwise or counterclockwise) of rotation. Since $S_{1}^{x}=$ $\pm \sqrt{1-\left(S_{1}^{y}\right)^{2}-\left(S_{1}^{z}\right)^{2}}$, it is then clear that, according to this sign it is always possible to rotate in such a way to have $\Delta E \leq 0$.

The same procedure can be applied $n$ times for any other $S_{i}^{z} \neq 0$, so that we will end with a configuration $\mathcal{C}^{(n)} \in \mathcal{A}$ (the rotation does not change the constraint) with energy $E^{(n)} \leq E_{\text {dis }}$. We can therefore consider the configurations in the $X Y$ plane. This choice has the main advantage that it is sufficient to consider as independent variables the angles $\theta_{i}$ of the $i$-th spin w.r.t. the $x$-axis, thus satisfying automatically the conditions on the unit spin modulus : $S_{i}^{x}=\cos \theta_{i}$ and $S_{i}^{y}=\sin \theta_{i}$. Therefore we have to minimize the following expression:

$H=\frac{1}{2} \sum_{j \neq i} c_{|i-j|}\left(\eta \cos \theta_{i} \cos \theta_{j}-\sin \theta_{i} \sin \theta_{j}\right) \equiv \eta H_{x}+H_{y}$

under the constraint $\sum_{i=1}^{N} \sin \theta_{i}=0$. Since

$E_{\text {dis }}=\operatorname{Min}\left(H \mid m_{y}=0\right) \geq \operatorname{Min}\left(\eta H_{x}\right)+\operatorname{Min}\left(H_{y} \mid m_{y}=0\right)$,

a lower bound of $E_{d i s}$ can be provided finding the minima of the two terms in the r.h.s. of Eq. (7). Note that 
the first term on the right of Eq.(7) does not contain constraints, indeed we will show in the next section that the absolute minimum of $H_{x}$ automatically satisfied the constraint $m_{y}=0$.

\section{B. Minimum of $H_{x}$}

The minimum of $H_{x}$ due to the overall plus sign $(\eta>$ $0)$, can be obtained as in a standard anti-ferromagnetic spin system with neighbors interaction, that is disposing alternatively the spins along the $x$-axis as +1 and -1 .

Indeed, for any $\alpha$, let us call the $k-t h$ spin component $S_{k}^{x}=s$ and rewrite the energy as follows:

$$
E_{x}=s \sum_{j \neq k} c_{|j-k|} S_{j}^{x}+\frac{1}{2} \sum_{k \neq i \neq j} c_{|k-i|} S_{k}^{x} S_{i}^{x} \equiv a s+b,
$$

where $a, b$ are constants independent of $s$. There are two possibilities, $a=0$ or $a \neq 0$. In the first case the energy $E_{x}$ turns out to be independent of the $k-t h$ spin, while for $a \neq 0$, the minimum is attained when $s$ has its maximal value $(+1$ if $a<0,-1$ if $a>0)$. So, in any case we can say that the minimum occurs when $|s|=1$. Since the procedure can be iterated for all spins components, the minimum occurs when $S_{k}^{x}= \pm 1$ for any $k$, namely in the class of Ising models $\left(\sigma_{1}, \ldots, \sigma_{N}\right)$ with $\sigma_{i}= \pm 1$ and long range interaction.

We still have to prove that the minimal energy is obtained when the spins have alternating signs. To this end, let us consider the interaction between two neighbor spin pairs, the $j$-th, namely $\sigma_{2 j-1} \sigma_{2 j}$ and the $(j+k+1)$-th, namely $\sigma_{2 j+2 k+1} \sigma_{2 j+2 k+2}$ (this can be done since there is an even number of spins). There are 16 possibilities but only six of them have different energy due to the symmetry on the whole change of sign. They are:

$$
\begin{array}{lll}
++ & ++ & E_{1}=2+c_{2 k+1}+2 c_{2 k+2}+c_{2 k+3} \\
++ & +- & E_{2}=c_{2 k+1}-c_{2 k+3} \\
++ & -+ & E_{3}=-E_{2} \\
++ & -- & E_{4}=2-c_{2 k+1}-2 c_{2 k+2}-c_{2 k+3} \\
+- & +- & E_{5}=-2-c_{2 k+1}+2 c_{2 k+2}-c_{2 k+3} \\
+- & -+ & E_{6}=-2+c_{2 k+1}-2 c_{2 k+2}+c_{2 k+3} .
\end{array}
$$

From the monotonicity of the function $c_{x}$ one gets,

$$
E_{5}<E_{1}, E_{2}, E_{3}, E_{4}
$$

Imposing $E_{5}<E_{6}$ we obtain

$$
2 c_{2 k+2}<c_{2 k+1}+c_{2 k+3}
$$

that holds from the convexity property of $c_{x}$.

Since $E_{5}$ is minimal for each pair interaction, the absolute minimum will be obtained using, for each pair, the configuration

$$
\mathcal{C}_{x}=\left\{S_{i}^{x}=(-1)^{i}\right\}_{i=1}^{N}
$$
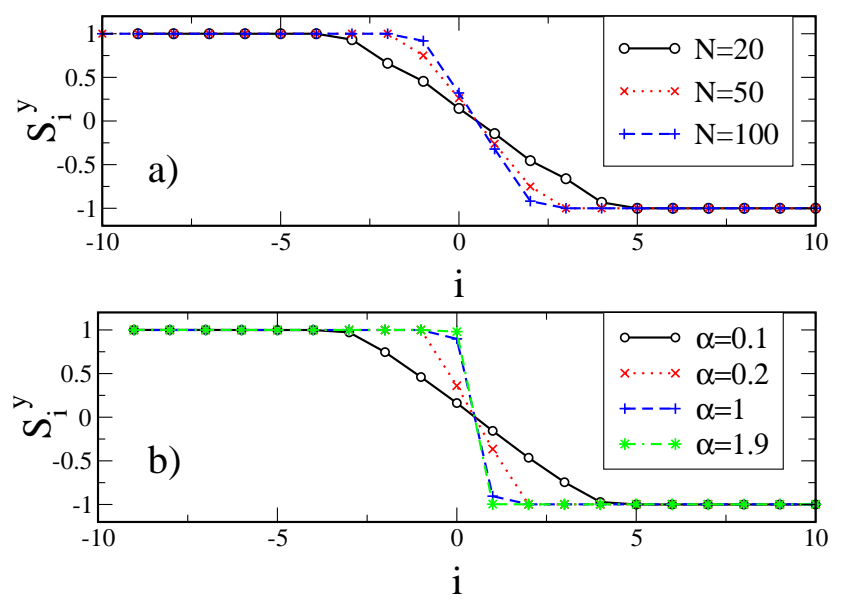

FIG. 1: (Color online) Spin values along the chain vs the spin index (only the central part of the chain has been shown) for the Hamiltonian $H_{y}$. a) for fixed $\alpha=0.1$ and different $N$ values as indicated in the legend; b) for fixed $N=20$ and different $\alpha$ values (see the legend).

The energy $E_{x}$ for this configuration can be computed immediately:

$$
E_{x}=\sum_{k=1}^{N-1}(-1)^{k} \frac{(N-k)}{k^{\alpha}} .
$$

Let us notice that such a result is far from being obvious. Indeed a decreasing non-convex function $c_{x}$ could give rise to a different minimal configuration.

\section{Minimum of $H_{y}$}

Let us now switch to the more difficult task (due to the constraint) of computing $E_{y}=\operatorname{Min}\left(H_{y} \mid m_{y}=0\right)$. Physically, due to the overall minus sign in front of $H_{y}$, one can expect that clusters of aligned spin with unit modulus (ferromagnetic order) will decrease the energy with respect to other configurations. This is surely true for nearest neighbor interaction $(\alpha=\infty)$ but it can not be true for all $\alpha$ values. For instance, when $\alpha=0$, the energy corresponding to the configuration with half spins equal to 1 and half equal to -1 (the order is irrelevant) is $E_{0}=N / 2>0$, while the true minimum $E=0$ is attained when all spins are 0 .

Then, the question arises of what can be the minimum in presence of a generic $\alpha$.

Applying the standard Lagrange multipliers formalism, one has to minimize the function:

$$
H=-\frac{1}{2} \sum_{j \neq i} c_{|i-j|} \sin \theta_{i} \sin \theta_{j}-\lambda \sum_{i} \sin \theta_{i} .
$$

where $\lambda$ is the Lagrange multiplier associated to $m_{y}=0$.

Taking the derivatives, we get, for each spin, two possible solutions:

$$
\cos \theta_{i}=0
$$




$$
\sum_{j \neq i} c_{|i-j|} \sin \theta_{j}-\lambda=0 .
$$

However, solving, even numerically, the system (1112) is more difficult than finding directly the minimum.

We have therefore calculated the minimal configuration under constraint, using an iterative optimization approach based on the FFSQP solver [14] and also developing the following approach outlined here below.

- Start with a random configuration with $m_{y}=0$.

- Chose for the $k-t h$ spin a new value between -1 to 1 and compute the energy. This generally produces a change in magnetization $\Delta m_{y} \neq 0$.

- Distribute equally $\Delta m_{y} \neq 0$ among the other spins taking into account the constraint about their modulus. Specifically subtract/add to every spin the minimum of its distance from the values \pm 1 and the mean of $\Delta m_{y}$.

- Iterate over all spins up to an energy variation less than some fixed value (from $10^{-3}$ to $10^{-8}$ in our simulations).

The two approaches give the same result: for any initial random configuration the algorithm described above converges to some smooth configuration, for any finite $N$ and $\alpha>0$, as indicated in Fig. 1 There, we considered respectively the case of $\alpha$ fixed varying $N$ (Fig. 1) and $N$ fixed varying $\alpha$ (Fig. 1b). Within the numerical errors the spins in the minimal energy configuration are distributed monotonically and anti-symmetrically w.r.t. the center of the chain. Then we assume that $E_{y}$ is given by an anti-symmetric distribution of the spin with a nondecreasing (or non increasing) monotonic dependence of the $y-$ spin component along the chain.

An interesting feature is the presence of a finite domain wall (defined by those spins having length less than 1) between two clusters with $S_{i}^{y}=+1(\uparrow)$ and $S_{i}^{y}=-1$ $(\downarrow)$ respectively. With decreasing range of interaction (increasing $\alpha$ : Fig. 1b) or increasing number of spins (Fig. 17), the interface region between the clusters $(\uparrow)$ and $(\downarrow)$ decreases. This agrees, at least qualitatively, with the results obtained for the nearest neighbor model $(\alpha=\infty)$ where the minimal configuration is given by

$$
\mathcal{C}_{\uparrow \downarrow}=(\uparrow \ldots \uparrow ; \downarrow \ldots \downarrow) .
$$

Thus, due to long range interaction, an interface region between the two clusters with opposite magnetization is produced. It is, of course, physically relevant to understand if the size of the interface region goes to zero in the $N \rightarrow \infty$ limit.

Strictly speaking the configuration $E_{\uparrow \downarrow}$ is not an absolute minimum for any $\alpha>0$ and finite $N$. In order to prove that, consider the configuration

$$
\mathcal{C}_{s}=(\uparrow \ldots \uparrow ; s ;-s ; \downarrow \ldots \downarrow),
$$

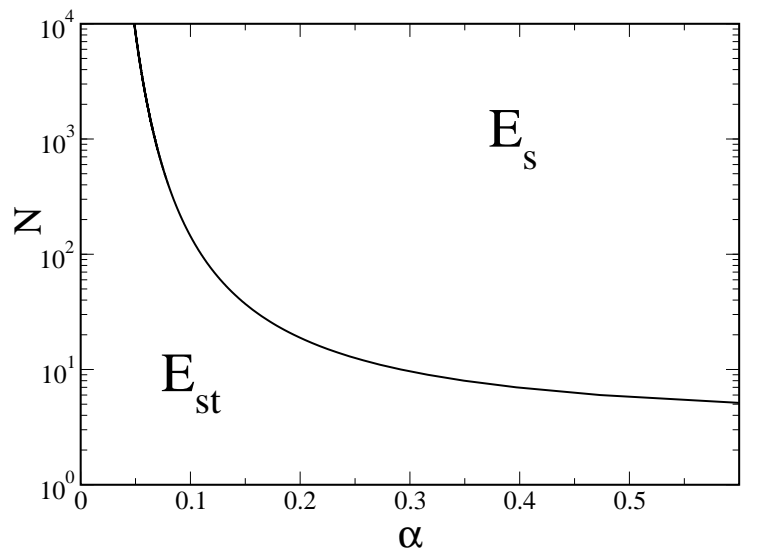

FIG. 2: Critical $N_{c r}$ as a function of $\alpha$. The region above the line is where the TNT is given by $E_{s}$ (one spin pair decreased) and the region below it is where the TNT has more than one spin pair decreased $\left(E_{s t}\right)$.

with $N-2$ spins satisfying condition (11) and the two central ones satisfying the condition (12). The energy $E_{s}$ corresponding to $\mathcal{C}_{s}$ can be written as

$$
E_{s}=\bar{E}+c_{1} s^{2}-2 s\left[c_{1}-c_{N / 2}\right],
$$

where $\bar{E}$ is independent of $s$. The minimum is thus obtained when $s=1-c_{N / 2} / c_{1} \neq 1$. The energy difference to $E_{\uparrow \downarrow}$ is

$$
\Delta E=E_{s}-E_{\uparrow \downarrow}=-\frac{c_{N / 2}^{2}}{c_{1}}=-\left(\frac{2}{N}\right)^{2 \alpha}<0 .
$$

Therefore, for any finite chain and finite $\alpha, E_{s}<E_{\uparrow \downarrow}$.

Physically, $\mathcal{C}_{s}$ has an energy less than $\mathcal{C}_{\uparrow \downarrow}$ due to border effects. Indeed the energy of two opposite spins of length 1 is $E=c_{1}$, while it is only $c_{1} s^{2}$ for two shorter spins $|s|<1$. On the other hand the interaction between $s(-s)$ and the other spins of length $1(-1)$ is canceled one to one but the interaction with the closest spin $\left(-s c_{1}\right)$ and with the last opposite one $\left(s c_{N / 2}\right)$. This gives Eq. (13).

The same procedure can be applied taking a trial configuration with energy $E_{s t}$ :

$$
\mathcal{C}_{s t}=(\uparrow \ldots \uparrow ; t ; s ;-s ;-t ; \downarrow \ldots \downarrow) .
$$

In this case a minimum with $s<t<1$ can be found only for $N<N_{c r}(\alpha)=\left(2^{\alpha+1}-1 / 2\right)^{1 / \alpha}$. Asymptotically, for large $N$, this implies that for $N>2 C^{1 / \alpha}$, where $C=2 e^{-1 / 4}>1$, the minimal solution has energy $E_{s}$. In Fig. 2 2 we show the graph of $N_{c r}(\alpha)$, and the two regions in the plane $(N, \alpha)$, where $E_{s}$ is the minimal solution, and where another minimal solution with four (or more) spins with length less than 1 is possible $\left(E_{s t}\right)$. Since $N_{c r}(\alpha) \rightarrow \infty$ for $\alpha \rightarrow 0$, for any $\alpha \neq 0$ a sufficiently large $N>N_{c r}(\alpha)$ value exists (thus in the thermodynamic limit) such that $E_{s}$ is the minimal solution. Then, for $N>N_{c r}(\alpha)$ :

$$
E_{y}=E_{\uparrow \downarrow}-\left(\frac{2}{N}\right)^{2 \alpha}
$$




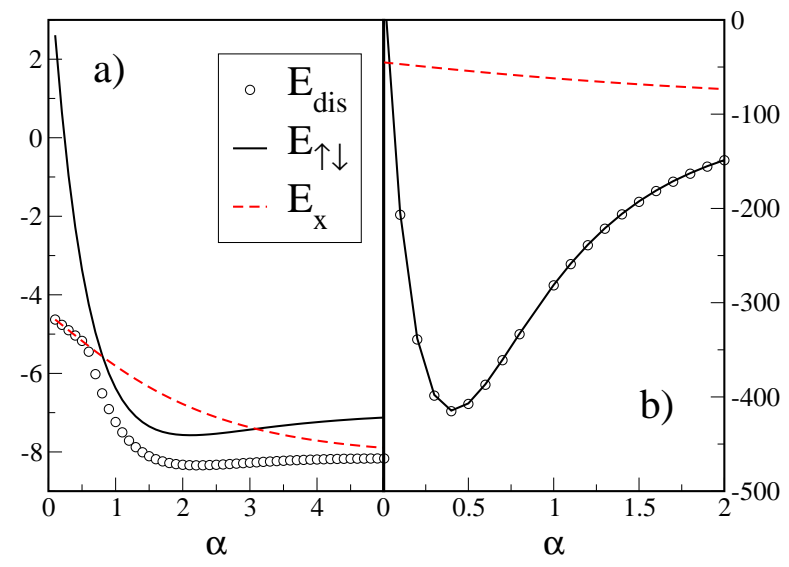

FIG. 3: (Color online) $E_{x}$ (full black line), $E_{\uparrow \downarrow}$ (dashed red line) and $E_{\text {dis }}$ (open circles) versus $\alpha$, for $\eta=0.9$ and different $N$ values : (a) $N=10$, (b) $N=100$.

where $E_{\uparrow \downarrow}$ can be written in closed form as:

$$
E_{\uparrow \downarrow}=\left(\frac{2}{N}\right)^{\alpha-1}+\sum_{k=1}^{N / 2-1} \frac{3 k-N}{k^{\alpha}}+\frac{N / 2-k}{(N / 2+k)^{\alpha}} .
$$

We thus proved that for $\alpha>0$ and $N>N_{c r}(\alpha)$

$$
E_{\uparrow \downarrow}>E_{d i s}>\eta E_{x}+E_{y}
$$

where the expressions for $E_{\uparrow \downarrow}, E_{x}$, and $E_{y}$ are given respectively by Eqs. (16, 90 15).

\section{Thermodynamic limit}

Let us now show that, in the long range case $0<\alpha<1$, the ratio $r$ between the disconnected ratio, defined by Eq. (4), goes to a non zero constant when the number of spins goes to infinity, while, for short range interaction $\alpha>1$, it goes to zero, thus revealing the intrinsic long range nature of the TNT.

The minimum energy, having as a configuration $\mathcal{C}_{\text {min }}=\left\{S_{y}^{i}=1\right\}_{i=1}^{N}$ (all spins aligned along the $\mathrm{y}$ direction) can be easily found:

$$
E_{\min }=\sum_{k=1}^{N-1} \frac{k-N}{k^{\alpha}} .
$$

Let us also define the quantities:

$$
\begin{aligned}
& r_{1}=\frac{E_{y}+\eta E_{x}-E_{\text {min }}}{\left|E_{\text {min }}\right|}, \\
& r_{2}=\frac{E_{\uparrow \downarrow}-E_{\text {min }}}{\left|E_{\text {min }}\right|} .
\end{aligned}
$$

Due to (17), $0<r_{1}<r<r_{2}$.

\section{Long Range}

Consider first the long-range case $0<\alpha<1$. The following asymptotic expression, for $N \rightarrow \infty$, can be found by substituting sum with integrals:

$$
\begin{aligned}
E_{\text {min }} & \simeq-\frac{N^{2-\alpha}}{(2-\alpha)(1-\alpha)}+O(N), \\
E_{\uparrow \downarrow} & \simeq N^{2-\alpha} \frac{1-2^{\alpha}}{(1-\alpha)(2-\alpha)}+O(N), \\
E_{x} & \simeq-b_{\alpha} N+O\left(N^{1-\alpha}\right),
\end{aligned}
$$

where $b_{\alpha}>0$ is a constant independent of $N$.

Since both $r_{1} \rightarrow\left|2-2^{\alpha}\right|$ and $r_{2} \rightarrow\left|2-2^{\alpha}\right|$ for $N \rightarrow \infty$, it follows $r \rightarrow\left|2-2^{\alpha}\right|$ too, so that the disconnected energy region remains finite w.r.t. the ES in the thermodynamic limit. This prove the disconnection of the system below the TNT. It is interesting to note that, as $\alpha \rightarrow 1, r \rightarrow 0$.

\section{Short Range}

In the short-range case, $\alpha>1$, one can write the following asymptotic expression (by substituting sums with integrals) :

$$
E_{\text {min }} \simeq c_{\alpha} N+O\left(N^{2-\alpha}\right),
$$

where $-1+1 /(1-\alpha)<c_{\alpha}<-1+2^{1-\alpha} /(1-\alpha)$.

Let us first show that, as $N \rightarrow \infty$ :

$$
\lim _{N \rightarrow \infty} \frac{E_{\uparrow \downarrow}-E_{\min }}{N}=0 .
$$

Computing explicitly the l.h.s. of (24) one gets :

$$
\begin{aligned}
& 0 \leq \lim _{N \rightarrow \infty} \frac{2}{N}\left(\sum_{k=1}^{N / 2-1} \frac{1}{k^{\alpha-1}}+\sum_{k=N / 2+1}^{N-1} \frac{N-k}{k^{\alpha}}\right) \\
& \leq \lim _{N \rightarrow \infty} \frac{2}{N}\left(\int_{1}^{N / 2} d x x^{1-\alpha}+\right. \\
& \left.\int_{N / 2+1}^{N} d x \frac{N-x}{x^{\alpha}}\right)=0 .
\end{aligned}
$$

Then, $r_{2} \rightarrow 0$ and, since $r_{2}>r>0$ it follows $r \rightarrow 0$ and the system is not disconnected.

This concludes our proof, which is valid for antisymmetric coupling (positive $\eta$ ).

\section{E. 1d, numerical solution for the full model}

Despite the proof of the existence of the TNT did not require the explicit knowledge of the spin configuration, it may be of some interest to find it.

Finding analytically the spin configuration of the full model under the constraint $m_{y}=0$ for any $\alpha, \eta$ and $N$ is 


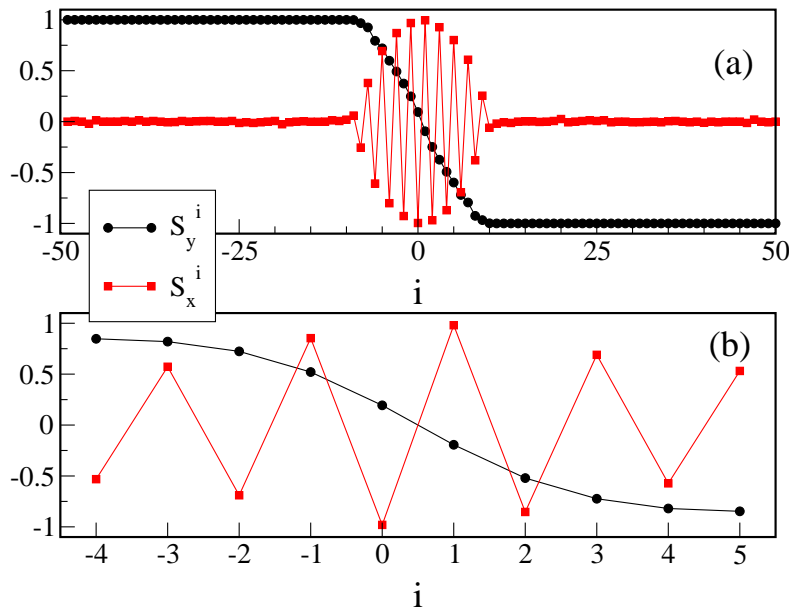

FIG. 4: (Color online) (a) $x$ and $y$ spin components for the numerical TNT. Here is $N=100, \eta=0.9$ and $\alpha=0.05$. (b) $x$ and $y$ spin components for the numerical TNT. Here is $N=10, \eta=0.9$ and $\alpha=\infty$.

a complicated task. Indeed, depending on the different values of the parameters, the minimal configuration can completely change its shape, for instance from all spins along the $x$-axis with alternating signs (giving rise to the energy $E_{x}$ ) to all spins along the $y$-axis (first half positive, second half negative) giving rise to $E_{\uparrow \downarrow}$. For instance, when $\alpha=0, E_{x}<0<E_{\uparrow \downarrow}$, while for $\alpha \rightarrow \infty$ and $N$ sufficiently large $E_{\uparrow \downarrow}<E_{x}<0$ (for small $N$ it is also possible to have $\left.E_{x}<E_{\uparrow \downarrow}<0\right)$.

This is explicitly shown in Fig. 3] where $E_{x}, E_{\uparrow \downarrow}$ and $E_{\text {dis }}$ (the TNT) obtained numerically for two different $N$ values have been plotted as a function of $\alpha$. As one can see, for $\alpha$ less than some value depending on $\eta$ and $N$, say $\alpha_{0}(N, \eta)$, one has $E_{x}<E_{\uparrow \downarrow}$, while for $\alpha>\alpha_{0}(N, \eta)$, one can have different possible situations depending on the $\eta$ and $N$ values. For instance for $N=10$ and $\eta=0.9$, $E_{x}<E_{\uparrow \downarrow}$ (Fig. [3]) for $\alpha \rightarrow \infty$, while $E_{\uparrow \downarrow}<E_{x}$ for $\alpha \rightarrow \infty$ and $N=100$ (Fig. $3 \mathrm{~b}$ ).

It is also instructive to describe the behavior of $E_{d i s}$ as a function of $\alpha$. As one can see (Fig. 3a), for relatively small $\alpha, E_{d i s}$ closely follows $E_{x}$ while for large $\alpha$ values, $E_{d i s}$, even if different from both, is closer to $E_{\uparrow \downarrow}$ than to $E_{x}$. This is the rule, at least for large $N$ values, and the difference between the configurations given by $E_{\uparrow \downarrow}$ and $E_{d i s}$ is only restricted to a small domain wall in the central part of the chain, see Fig. 4a, where the configuration $\mathcal{C}_{d i s}$ in a long range case has been shown. Completely different is the situation for small $N$ values, e.g. Fig. 4b. Here only 10 particles are considered. In this case, for $\alpha \rightarrow \infty$ (see discussion above) $E_{x}<E_{\uparrow \downarrow}$ and the configuration $\mathcal{C}_{d i s}$ is something between $\mathcal{C}_{x}$ and $\mathcal{C}_{\uparrow \downarrow}$ (see Fig. 4 p.)

Let us analyze in detail the behavior of the domain wall under a change in the parameters of the system. To this end we define the energy domain as

$$
E_{\text {domain }}=\left|E_{\text {dis }}-E_{\uparrow \downarrow}\right| .
$$

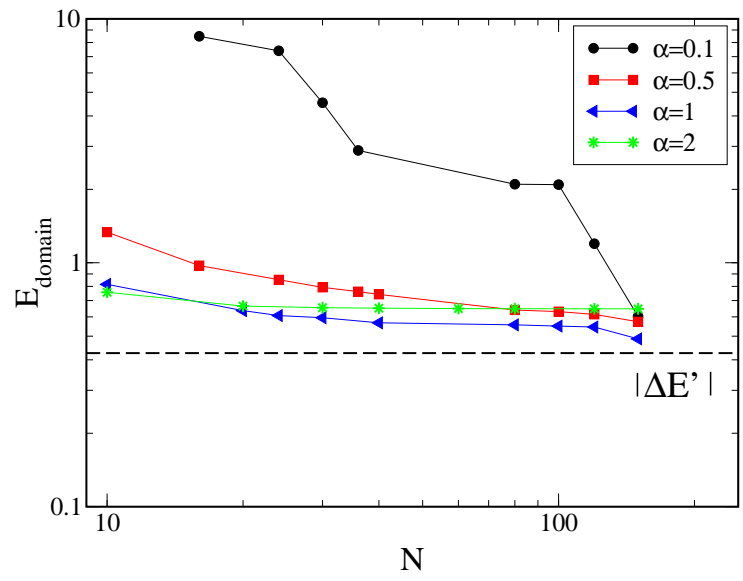

FIG. 5: (Color online) Domain wall energy as a function of $N$ for different $\alpha$ values, as indicated in the legend, and $\eta=0.9$. Also shown as horizontal dashed line $\left|\Delta E^{\prime}\right|=\eta^{2} /(1+\eta)$.

Its behavior for different $N$ and $\alpha$ values has been shown in Fig. [5] As one can see, in the large $N$ limit, the energy domain approaches, for any $\alpha$, some finite nonzero value dependent only of $\eta$. This is remarkably different from the domain wall obtained by minimizing $H_{y}$ under the constraint $m_{y}=0$, see Sec. IIIC where the formation of the domain wall was essentially due to border effects and whose energy disappears for large $N$ values, see Eq. (15).

This asymptotic value can be understood as follows: consider the trial configuration

$\mathcal{C}_{x y}=\left\{\begin{array}{l}S_{x}^{i}=\left\{0, \ldots, 0,+\sqrt{1-s^{2}},-\sqrt{1-s^{2}}, 0, \ldots, 0\right\} \\ S_{y}^{i}=\{1, \ldots, 1,+s,-s,-1, \ldots,-1\} .\end{array}\right.$

The energy $E^{x y}(s)$ of this configuration is given by

$$
E^{x y}(s)=\bar{E}+c_{1} s^{2}-2 s\left[c_{1}-c_{N / 2}\right]-\eta c_{1}\left(1-s^{2}\right),
$$

where $\bar{E}$ is independent of $s$ and $\eta$.

The minimum, as a function of $s$, is

$$
s_{\min }=\frac{c_{1}-c_{N / 2}}{c_{1}(1+\eta)}
$$

Then, for $N \rightarrow \infty, s_{\text {min }} \rightarrow 1 /(1+\eta)$, which is independent from $\alpha$. This value can be compared with our numerical results. The energy difference to $E_{\uparrow \downarrow}$ in the limit $N \rightarrow \infty$ is:

$$
\Delta E^{\prime}=E^{x y}\left(s_{\min }\right)-E_{\uparrow \downarrow}=-\frac{\eta^{2}}{1+\eta}<0 .
$$

Its absolute value has been indicated as a horizontal dashed line in Fig. 5 As one can see, all curves are close to $\left|\Delta E^{\prime}\right|$ even at $N \sim 100$. While we cannot exclude that other configurations, with four or more central spins $S_{i}^{y}<1$, have an energy less than $E^{x y}\left(s_{\min }\right)$, we surely have found a minimal configuration whose energy is differing from $E_{\uparrow \downarrow}$ for a finite quantity in the limit $N \rightarrow \infty$. 


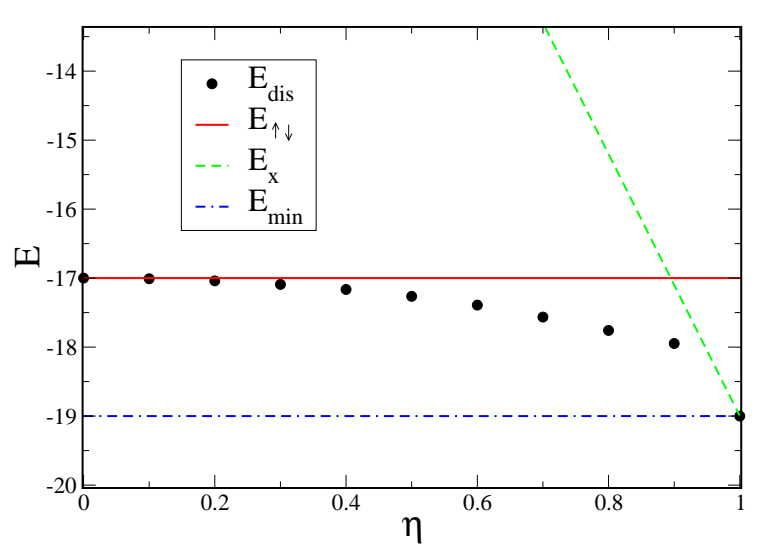

FIG. 6: (Color online) TNT for the nearest neighbor interaction $(\alpha=\infty)$ vs the parameter $\eta$. Here is $N=20$. Different energies are indicated in the caption inside the figure.

This in turn implies that the domain does not disappear in the thermodynamic limit.

As a last remark, let us stress that the no-disconnection of a system does not necessarily imply the non existence of a TNT different from the minimal energy at finite $N$, even for short range interaction. To this end we consider the strongest short range coupling, namely the nearest neighbor one $(\alpha=\infty)$ and compute numerically the TNT. Results are presented in Fig. (6).

As one can see the numerically computed $E_{d i s}$ is different from $E_{\text {min }}$ for $\eta \neq 1$ so that a finite range of energies $E_{\text {min }}<E<E_{d i s}$ exists for finite $N$ and nearest neighbor interaction. Increasing $N$, the size of this energy range remains constant, while $E_{\min } \sim N$. That is why the ratio $r \rightarrow 0$ for large $N$ values. From the same Fig. [6] it is clear that $\mathcal{C}_{\text {dis }}$ goes continuously from a configuration close to $\mathcal{C}_{\uparrow \downarrow}$ when $\eta \ll 1$ to one close to $\mathcal{C}_{x}$ when $\eta \simeq 1$.

\section{MULTIDIMENSIONAL CASE}

The results obtained in the previous Sections for $d=1$ can be extended in greater dimension $d \geq 2$.

While it can be easily shown that, in the short-range case $d<\alpha$, the system cannot be disconnected in the thermodynamic limit, the proof of the disconnection for the long-range case is essentially based on the assumption that the minimum energy with the constraint $m_{y}=0$ is given by an obvious extension of what we have found in $d=1$. This is, at the moment, justified only by our numerical simulations.

Let us then consider a $d$-dimensional hypercube of side $L$, such as $L^{d}=N$ and divide it in two equal halves. Let us then put half of the spins with $y$-component in one region and the other half in the remaining with opposite $y$-component and call $E_{\uparrow \downarrow}$ the resulting energy for such configuration. Surely the TNT has an energy value less or equal to $E_{\uparrow \downarrow}$, that is $E_{d i s} \leq E_{\uparrow \downarrow}$.
$2 \mathrm{D}$
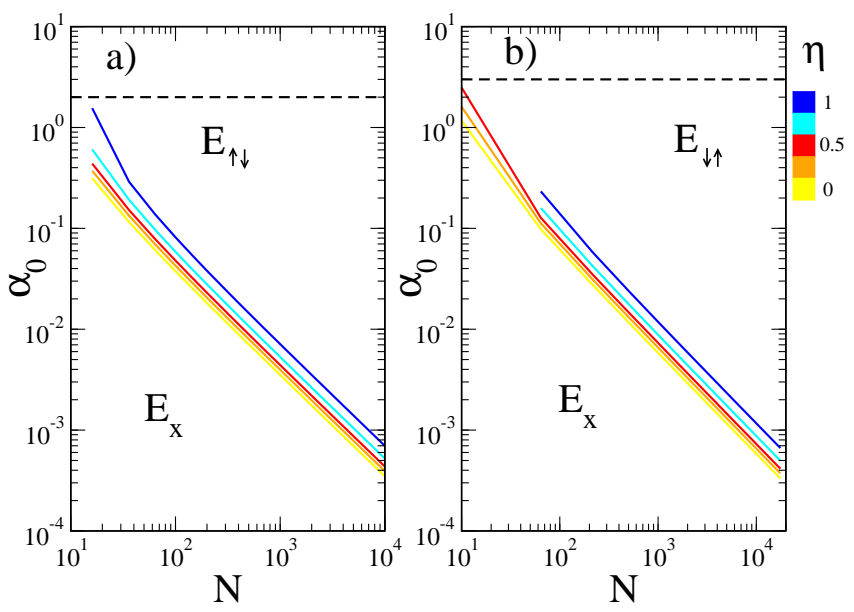

FIG. 7: (Color online) Critical $\alpha_{0}$ as a function of the number of spins for different $\eta$ indicated by different colors. (a) $d=2$, (b) $d=3$.

Let us then write:

$$
\begin{aligned}
E_{\text {min }} & =E_{\uparrow}+E_{\uparrow}+V_{\uparrow \uparrow}, \\
E_{\uparrow \downarrow} & =E_{\uparrow}+E_{\downarrow}+V_{\uparrow \downarrow},
\end{aligned}
$$

where $E_{\uparrow}, E_{\downarrow}$ are the energies of the respective halves and $V_{\uparrow \downarrow}, V_{\uparrow \uparrow}$ are the interaction energies between the two halves with, respectively, antiparallel and parallel spins.

Since $E_{\uparrow}=E_{\downarrow}$ and $-V_{\uparrow \uparrow}=V_{\uparrow \downarrow}>0$, one has:

$$
0 \leq r \leq r_{\uparrow \downarrow}=2 \frac{V_{\uparrow \downarrow}}{\left|E_{\text {min }}\right|}=2 \frac{2 E_{\uparrow}-E_{\text {min }}}{\left|E_{\text {min }}\right|} .
$$

We will make use of the results found in Ref. 15], that in our variables read as:

$$
\lim _{N \rightarrow \infty} \frac{E_{\min }(d, \alpha, N)}{N^{2-\alpha / d}-N}=C_{d}(\alpha),
$$

for $\alpha \neq d$ and where the constant $C_{d}(\alpha)>0$ for $d<\alpha$ and $C_{d}(\alpha)<0$ for $d>\alpha$ depends only on $d$ and $\alpha$.

\section{Short-Range}

Let us discuss the short range case $\alpha>d$. In this case we have,

$$
\lim _{N \rightarrow \infty} \frac{E_{\min }(d, \alpha, N)}{N}=C_{d}(\alpha),
$$

and, since $E_{\uparrow}=E_{\min }(d, \alpha, N / 2)$, we can write

$$
0 \leq r \leq r_{\uparrow \downarrow}=2 \frac{2 E_{\uparrow} / N-E_{\min } / N}{\left|E_{\min } / N\right|} \rightarrow 0, \text { for } N \rightarrow \infty .
$$

This proves that, in the short-range case, $r \rightarrow 0$ for $N \rightarrow \infty$. 


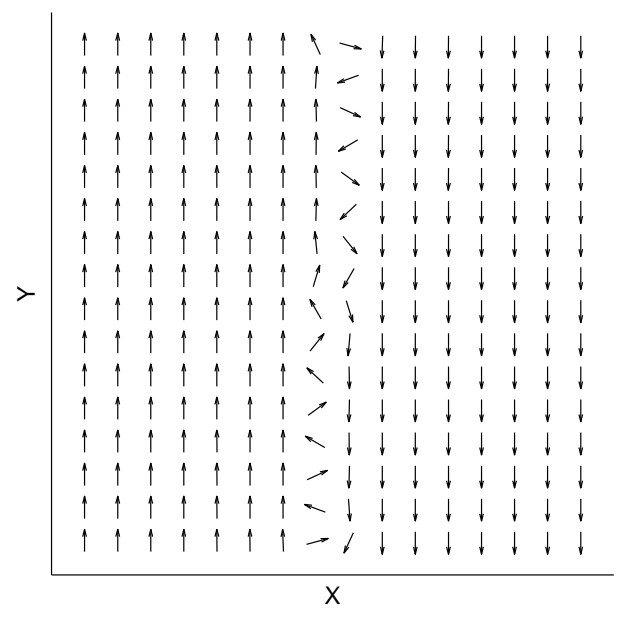

FIG. 8: (Color online) TNT for the 2-d square lattice, for the case $\alpha=0.1, \eta=0.5, N=16 \times 16=256$ spins. Here is $E_{\text {dis }}=-655.968997$, while $E_{\uparrow \downarrow}=-654.7308$.

\section{Long-Range}

In the long range-case, $\alpha<d$, let us assume that, for large $N$ values, $E_{\text {dis }} \rightarrow E_{\uparrow \downarrow}$.

Estimate (33) becomes in this case:

$$
\lim _{N \rightarrow \infty} \frac{E_{\min }(d, \alpha, N)}{N^{2-\alpha / d}}=C_{d}(\alpha)
$$

so that, for $N \rightarrow \infty$,

$$
r \simeq r_{\uparrow \downarrow}=2 \frac{2 E_{\uparrow} / N^{2-\alpha / d}-E_{\min } / N^{2-\alpha / d}}{\left|E_{\min } / N^{2-\alpha / d}\right|} \rightarrow 2-2^{\alpha / d}
$$

That way, $r \rightarrow$ const $\neq 0$ for $N \rightarrow \infty$ and $\alpha \neq d$, and a finite disconnected energy range exists in the thermodynamic limit.

It is also interesting to note that, as $\alpha \rightarrow d, r \rightarrow 0$, so that the result (35) is recovered.

The disconnection of the system in the long range case can thus be proved if we assume $\mathcal{C}_{\text {dis }} \sim \mathcal{C}_{\uparrow \downarrow}$.

Numerical simulations confirm this assumption. Indeed, let us define $\alpha_{0}(\eta, N)$ as the smallest value such that,

$$
E_{x}\left(\alpha_{0}, \eta, N\right)=E_{\uparrow \downarrow}\left(\alpha_{0}, \eta, N\right) .
$$

Its general dependence on parameters has been presented in Fig. 7 for $d=2,3$. As one can see, $\alpha_{0} \sim 1 / N \rightarrow 0$ when $N \rightarrow \infty$. In the same picture we indicate the regions where $E_{x}$ or $E_{\uparrow \downarrow}$ are respectively the minimal energies satisfying the constraint $m_{y}=0$. Also, $\alpha_{0}=d$ is plotted as a dashed horizontal line, showing that the short-range case (above the line) is characterized by $E_{\uparrow \downarrow}$, while the long-range case (below the line) can have different behaviors $\left(E_{x}\right.$ or $\left.E_{\uparrow \downarrow}\right)$, even if physically interesting long-range interactions are generally characterized by $E_{\uparrow \downarrow}$.

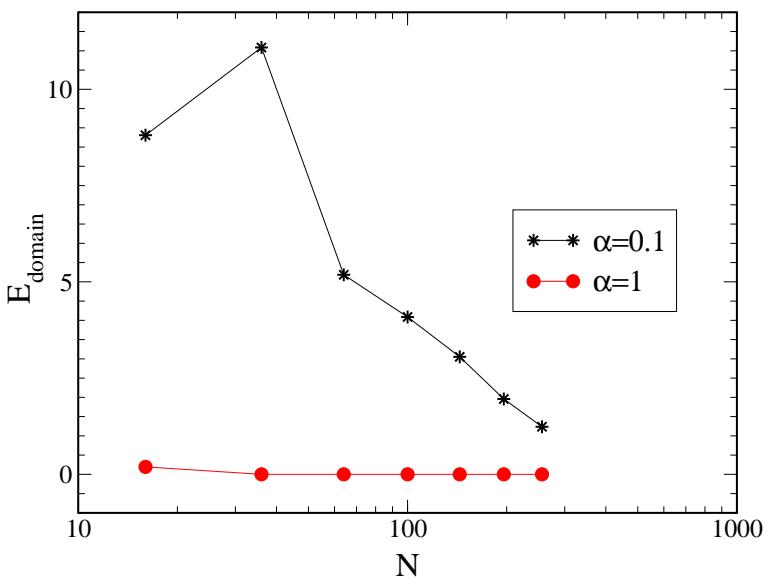

FIG. 9: (Color online) Domain energy as a function of the number of lattice spins, for the 2 -d square lattice and $\eta=0.5$, asterisks $(\alpha=0.1)$, circles $(\alpha=1)$. For $\alpha>1, E_{\text {domain }}$ becomes smaller than the computer precision.

As for the spin configuration $\mathcal{C}_{d i s}$, both for $d=2$ and $d=3$, all physically significant cases can be represented by $\mathcal{C}_{\uparrow \downarrow}$. Deviations occur for $\alpha<<d$, where a domain wall appears, see for instance Figs. [8] As one can see, $\mathcal{C}_{\text {dis }}$ is generically represented by two macroscopic blocks, with opposite sign of the $y$-magnetization, with a domain wall at their interface. In the domain wall the $y$ components increase in absolute value toward the center and the $x$-components are more or less distributed with alternating signs, see Figs. 8

Defining the domain energy, as the difference between the numerically found TNT and the energy $E_{\uparrow \downarrow}$ (26), one has that with increasing $N$ it goes to some constant or zero value (see Fig. 9) so that, in the thermodynamic limit $E_{d i s} \sim E_{\uparrow \downarrow}$, which justify, at least numerically, our previous assumption. This concludes our proof of the disconnection of the system with $\eta \geq 0$ for long-range interaction in any dimension $d$.

\section{NEGATIVE $\eta$}

In this last part we briefly discuss the case $\eta<0$. First of all $\eta>0$ is not a necessary condition for the existence of a finite disconnection region.

Let us first consider the $1-\mathrm{d}$ case; In Eq. (7) $\eta H_{x}$ becomes ferromagnetic as $H_{y}$, and the configuration

$$
\mathcal{C}_{x}^{\prime}=\left\{S_{x}^{i}=1\right\}_{i=1}^{N} \in \mathcal{A},
$$

has an energy $E_{x}^{\prime}<E_{x}$ (which is the energy of the configuration $\mathcal{C}_{x}$, see Eq. (8) ). Moreover, since the number of parallel spins in $\mathcal{C}_{x}^{\prime}$ is larger than in $\mathcal{C}_{\uparrow \downarrow}$, we will expect that the energy $E_{x}^{\prime}$, even if decreased by a factor $\eta$, will become soon or later less than $E_{\uparrow \downarrow}$.

While this has no consequences in the case $\alpha>1$, (we still have $r_{2} \rightarrow 0$ and the TNT does not exist), in the 


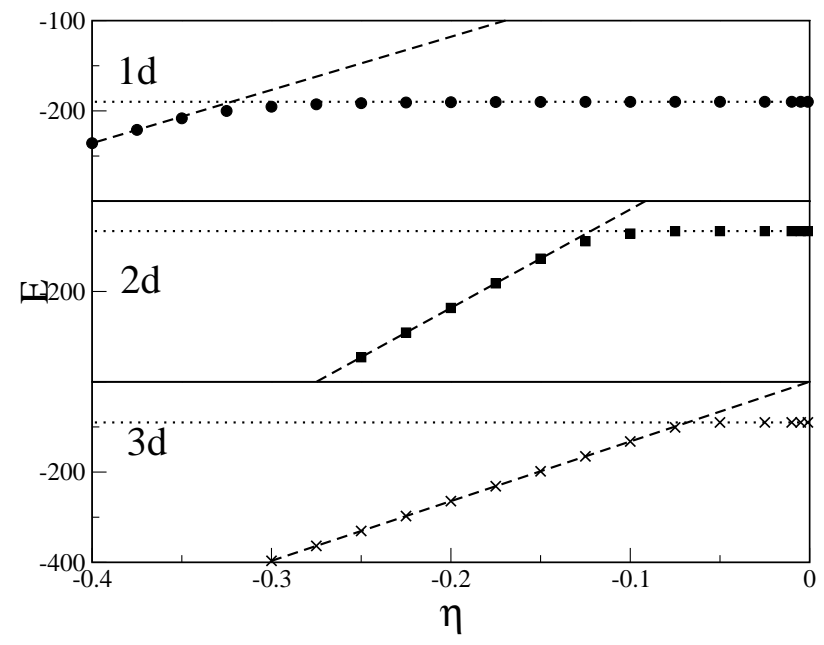

FIG. 10: $E_{\text {dis }}$ (symbols) as a function of $\eta$ for the long range case $\alpha=0.5$ and $N=64(d=1), N=8 \times 8(d=2)$, $N=4 \times 4 \times 4(d=3)$. Also shown as dotted horizontal lines $E_{\uparrow \downarrow}$, and, as dashed transverse lines $E_{x}^{\prime}$.

long-range case $(0<\alpha<1)$ some interesting features appear.

From Eq. (19) one has $r_{1} \rightarrow\left|2+\eta-2^{\alpha}\right| / 2$ for $N \rightarrow \infty$ and a finite disconnection energy region still exists for $2^{\alpha}-2<\eta<0$.

In the other case $-1<\eta<2-2^{\alpha}$ nothing can be said, even if, according to our numerical simulations $\mathcal{C}_{d i s} \sim \mathcal{C}_{x}^{\prime}$. This effectively happens in all dimensions $d=1,2,3$, as indicated in Fig. [10] where $E_{d i s}$ as a function of $\eta$ as been shown in a long range case. As one can see $E_{\text {dis }}$ is close to $E_{x}^{\prime}$ (equal, within numerical accuracy, according to our simulations) for $\eta<\eta_{c r}(\alpha, N)$, while it becomes close to $E_{\uparrow \downarrow}$, for $\eta>\eta_{c r}(\alpha, N)$. That holds true in any dimensions.

Let us note that, as a realistic $\eta_{c r}$, we can assume the intersection point between $E_{x}^{\prime}$ and $E_{\uparrow \downarrow}$, see Fig. (10). An estimate, that holds only in the thermodynamic limit can be obtained following the considerations made in Sec. (IV) giving $\eta_{c r} \sim 1-2^{\frac{\alpha}{d}}$.

It is also clear that assuming:

$$
E_{d i s}=\eta E_{x}=-\eta E_{\text {min }}
$$

one has $r \rightarrow 1+\eta$, and the system is disconnected even for negative $\eta$ in all dimensions.

\section{CONCLUSIONS}

Summarizing, we have studied the occurrence of a topological non-connectivity threshold (TNT) in anisotropic Heisenberg models in $d=1,2,3$ with an interaction strength depending on a power law of their relative distance with the exponent $\alpha$. We have found that the system, in the thermodynamic limit, is disconnected only in presence of a long-range interaction $0 \leq \alpha<d$. On the other side, in the short-range case, the ratio between the disconnected energy region and the total energy region goes to zero when $N \rightarrow \infty$. The anisotropy represents in this class of systems a necessary condition : indeed, in the isotropic case, the TNT coincides with the minimal energy, thus there is no disconnected energy region.

Future investigations concern the experimental evidence of TNT, for instance by looking for the divergence of de-magnetization times [9] as a function of energy in small magnetic samples.

Finally, let us point out that from a quantum mechanical point of view the classical disconnection does not exclude the flipping of the magnetization through Macroscopic Quantum Tunneling [16]. Thus the existence of TNT could give the possibility to study the emergence of Macroscopic Quantum Phenomena in a wide energy range (for macroscopic long range interacting systems), as has been shown in [17], where the quantum signatures of the TNT in an anisotropic Heisenberg model with allto-all interaction have been studied, and the relevance of the TNT w.r.t. Macroscopic Quantum Phenomena addressed.

\section{ACKNOWLEDGMENT}

We acknowledge useful discussion with J.Barre, F.M.Izrailev, R.Loubere, D.Mukamel and S.Ruffo.
[1] F. Borgonovi, G. L. Celardo, M. Maianti, E. Pedersoli, J. Stat. Phys., 116, 1435 (2004)

[2] D. Ruelle, Helvetica Physica Acta, 36, 183 (1963).

[3] J. Barré, D. Mukamel, S. Ruffo, Phys. Rev. Lett. 87, 3, (2001)

[4] N. Goldenfeld, Lectures on Phase Transitions and the Renormalization Group Addison-Wesley, Reading, MA (1992).

[5] D.J. Amit Modeling Brain Functions, Cambridge University Press, Cambridge, UK (1989).

[6] P.J. Ford, Contemp. Phys. 23, 141 (1982).
[7] A. Campa, A. Giansanti and D. Moroni, Phys. Rev. E 62, 303 (2000); F. Tamarit and C. Anteneodo, Phys. Rev. Lett. 84, 208 (2000).

[8] R.G. Palmer, Adv. in Phys., 31, 669 (1982).

[9] G.L. Celardo, J. Barré, F. Borgonovi and S. Ruffo, cond-mat/0410119

[10] L. Casetti, M. Pettini, and E.G.D. Cohen, J. Stat. Phys. 111, 1091 (2003).

[11] L.Q. English et al., Phys. Rev. B ,67, 24403 (2003); M. Sato et al., Jour. of Appl. Phys., 91 , 8676 (2002).

[12] P. Bruno, Phys. Rev. B 39, 865 (1989); D. Weller et al, 
Phys. Rev. Lett. 753752 (1995); J. Dorantes-Davila and G.M. Pastor, Phys. Rev. Lett. 81, 208 (1998); M. Pratzer et al, Phys. Rev. Lett. 87, 127201 (2001).

[13] R.M. White, "Quantum Theory of magnetism", McGRAW-HILL (1970).

[14] J.L. Zhou, A.L. Tits and C.T. Lawrence, User's Guide for FFSQP Version 3.7, Systems Research Center TR-92107r2, University of Maryland, College Park, MD (1997).

[15] S.A. Cannas and F.A. Tamarit, Phys. Rev. B, 54, R12661
(1996); T. Dauxois, S. Ruffo, E. Arimondo, M. Wilkens Eds., Lect. Notes in Phys., 602, Springer (2002).

[16] E.M. Chudnovsky and J. Tejada, Macroscopic Quantum Tunneling of the Magnetic Moment, Cambridge University Press, (1998).

[17] F. Borgonovi, G.L. Celardo and G.P. Berman, cond-mat/0506233 